\title{
Association between sarcopenia with lifestyle and family function among community-dwelling Chinese aged 60 years and older
}

Shan Hai, Hui Wang, Li Cao, Ping Liu, Jianghua Zhou, Ying Yang and Birong Dong*

\begin{abstract}
Background: Sarcopenia is defined as the age-related decline in skeletal muscle mass and function. The risk factors and causes of sarcopenia must be identified to develop prevention and treatment strategies for this syndrome. Our aim was to examine the association between sarcopenia with lifestyle and family function among community-dwelling Chinese people aged 60 years and older.

Methods: We conducted this study to evaluate sarcopenia among 834 community-dwelling Chinese individuals aged $\geq 60$ years using the Asian Working Group for Sarcopenia (AWGS) criteria. The sociodemographic characteristics, food consumption patterns, habits of smoking, and alcohol consumption of the participants were collected using a general questionnaire, whereas physical activity was assessed using the International Physical Activity Questionnaire (IPAQ; long-form version). Family function was assessed using the Family APGAR scale. In addition, the association of sarcopenia with lifestyle and family function was examined using univariate and multivariate analyses.
\end{abstract}

Results: The total prevalence rate of sarcopenia was 10.6\%. Female participants with sarcopenia had a lower frequency per week of nut consumption than those without sarcopenia $(p<0.05)$, whereas, for male participants, the differences in food consumption patterns of those with sarcopenia versus those without sarcopenia were not significant. Among the participants, the mean Family APGAR score was 8 (standard deviation $[S D]=0.92$ ). For both sexes, participants with sarcopenia had lower family function scores than those without sarcopenia. In the multivariate model, after adjustment for all covariates, frequency per week of nut consumption (adjusted OR $0.724,95 \% \mathrm{Cl} 0.532-0.985, P<0.05$ ) and Family APGAR score (adjusted OR $0.683,95 \% \mathrm{Cl} 0.496-0.940, P=0.019$ ) were statistically significantly associated with sarcopenia. The relationship between other lifestyle habits and sarcopenia was not significant.

Conclusion: There was significant association between sarcopenia with intake of nuts and family function. Further studies should evaluate if adequate intake of nuts and a well-functioning family may be effective in lowering the risk of sarcopenia.

Keywords: Chinese, Community, Family APGAR scale, Sarcopenia

\footnotetext{
* Correspondence: dongbirong33@163.com

Department of Geriatrics, West China Hospital, Sichuan University, 37 Guoxue Lane, Chengdu, NO 610041, China
} International License (http://creativecommons.org/licenses/by/4.0/), which permits unrestricted use, distribution, and reproduction in any medium, provided you give appropriate credit to the original author(s) and the source, provide a link to the Creative Commons license, and indicate if changes were made. The Creative Commons Public Domain Dedication waiver (http://creativecommons.org/publicdomain/zero/1.0/) applies to the data made available in this article, unless otherwise stated. 


\section{Background}

Sarcopenia is defined as the age-related decline in skeletal muscle mass and function [1], which may result in falls, physical disabilities, fracture, loss of dependence, poor quality of life and increased risk of death in older people [2]. Sarcopenia is also a common health problem that is associated with a high personal consumption and increased medical costs. The health care cost of sarcopenia in the United States alone was estimated at 18.5 billion dollars in the year of 2000 [3]. Therefore, reducing the incidence of sarcopenia and improving health-related quality of life in patients with sarcopenia are becoming increasingly important areas of research.

The risk factors and causes of sarcopenia must be identified to develop prevention and treatment strategies for this disease, particularly concerning lifestyle habits, which are more controllable in comparison to agerelated systemic changes and genetic factors. According to several recently published studies, a diet characterized by an adequate intake of protein, a high intake of essential amino acids (EAA), particularly leucine-rich food such as beef, fish, and legumes and supplementation of vitamin $\mathrm{D}$, is associated with a reduced risk for developing sarcopenia [4-6]. However, other lifestyle habits such as chronic alcohol consumption and smoking may promote loss of muscle mass and strength in old age indicating that they may be risk factors for sarcopenia $[7,8]$. Some cross-sectional and prospective studies have demonstrated that higher levels of physical activity have positive effects on muscle mass or strength $[9,10]$. However, findings from other studies have conflicted regarding whether or not lower self-reported physical activity is associated with a greater likelihood of developing sarcopenia [11]. Currently, a majority of studies on the association between sarcopenia and lifestyle are from Western countries. However, limited studies have reported on the prevalence and risk factors of sarcopenia in Chinese adults, particularly in community-dwelling individuals.

Family function has been found to be associated with health conditions of the elderly in several studies $[12,13]$. For example, many elderly adults with chronic health conditions have severe family dysfunction, and poor family function scores are correlated with the presence of chronic illness [14]. For most elderly Chinese adults, families are their primary source of social support, which is a recognized determinant of health. However, presently, fewer studies have focused on the relationship between sarcopenia and family function.

Because genetic background, ethnicity, and living environment differ from other countries among the Chinese population, the precise factors associated with sarcopenia must be defined. Thus, the aim of this study was to determine the association of sarcopenia with lifestyle and family function among the community-dwelling population aged $\geq 60$ years.

\section{Methods \\ Study design}

We used various advertising strategies mostly including displaying posters to recruit volunteers in the Wuhou District, Chengdu, Sichuan province in southwest China on convenience sampling basis. Individuals aged $\geq 60$ years who lived in the community for more than 12 months were recruited for the study. Participants with the following conditions were excluded from the study: (1) physical disabilities such as loss of hand or foot or limbs; (2) an implanted electronic device or orthopedic metal implantations. (3) unable to perform the handgrip strength test or walking test;(4)dementia and therefore, unable to communicate with interviewers; or (5) later stages of malignancies with cachexia; A total of 848 persons aged $\geq 60$ years were recruited from the community population of Chengdu from August 2014 to July 2015. Of these participants, 14 were excluded from the study because of missing data. We collected and analyzed data for 834 participants (415 men and 419 women).

Trained personnel visited all the participants in person and collected the data. Sociodemographic characteristics including educational level, age, sex and occupation were recorded using a general questionnaire. Cognitive function was measured with the 30-item MMSE, a global test with the following components: attention, orientation, language, calculation, and recall. (sensitivity 80-90\%, specificity 80-100\%) [15]. We assessed depression using short form 15-items Chinese version of Geriatric Depression Scale (GDS-15) [16]. The prevalence of specific medical conditions was established using standardized criteria that combined the history of the physical illness, diagnosis, and corresponding medication. The anthropometric variables of all participants were checked. Body weight was measured in light clothing to the nearest $0.01 \mathrm{~kg}$, and height was measured to the nearest $0.01 \mathrm{~cm}$. Body mass index (BMI) was calculated as weight in kilograms divided by height squared in meters $\left(\mathrm{kg} / \mathrm{m}^{2}\right)$.

\section{Collection of information on lifestyle}

We collected data on lifestyle. Lifestyle criteria included dietary patterns, physical activity, and smoking and drinking habits. A validated simplified FFQ was used for assessing dietary information. All participants completed the questionnaire about the frequency of eating each food item and the frequency units (day, week, month, or never) based on nine food categories: grain (or cereals), vegetables, fruit, meat (pork, beef, poultry, and mutton), eggs, fish and shrimp, milk and milk products, legumes and nuts. The nine kinds of foods were based on the Chinese Food Guide Pagoda, which is extensively used 
for the dietary guidelines of Chinese residents. Each food category frequency was converted into times per week (times/week). The validity and reliability of FFQ have been described elsewhere [17]. Participants were classified as current smokers, past smokers and never smoker. Subjects who had smoked everyday in the past year were classified as current smokers, whereas those who had smoked every day for more than 2 years in the year before the study began were classified as past smokers. They were also classified by their consumption of alcohol into no-drinkers, low-risk drinkers, and high-risk drinkers. High-risk drinking was defined as drinking more than twice a week. Those who consumed alcohol less than twice a week were classified as low-risk drinkers. The questionnaire had yes or no items only. Physical activity was assessed using a Chinese version of the International Physical Activity Questionnaire (IPAQ; long form) [18].

\section{Assessment of family function}

Family function was assessed using the Family APGAR score. The Family APGAR scale, designed by Smilkstein in 1978, is used to evaluate an individual's satisfaction with their perceived level of family function [19]. It consists of five parameters: Adaptability, Partnership, Growth, Affection, and Resolve. A three-point scale ranging from 0 (hardly ever) to 2 (almost always) is used to score all the items. The total score range varies from 0 to 10 . Higher scores indicate higher levels of satisfaction with family functioning. A score of $0-3$ indicates severe family dysfunction, 4-7 moderate family dysfunction, and 8-10 good family function. It has been used widely in China, with satisfactory validity and reliability $[20,21]$.

\section{Assessment of sarcopenia}

We defined sarcopenia, according to the AWGS criteria, [2] as the presence of low muscle mass, plus low muscle strength, or low physical performance.

Muscle mass was measured using bioelectrical impedance analysis (BIA) (Maltron BioScan 920 II, Rayleigh, UK). Appendicular skeletal muscle mass (ASM) was calculated as the sum of skeletal muscle in the arms and legs. The height-adjusted appendicular skeletal muscle mass (ASMI) was defined as ASM divided by height squared in meters (ASM/height ${ }^{2}$ ). Low muscle mass was defined as a height-adjusted ASM of less than $7.0 \mathrm{~kg} / \mathrm{m}^{2}$ in men and $5.7 \mathrm{~kg} / \mathrm{m}^{2}$ in women [2]. Muscle strength was assessed by grip strength and measured using a dynamometer (CAMRYEH 101, China). Participants exert maximum effort three times with each hand. Three trials for each hand were carried out and we used the highest value for diagnosing sarcopenia. Low handgrip strength was defined as $<26 \mathrm{~kg}$ in men and $<18 \mathrm{~kg}$ in women, using the AWGS consensus cutoff points [2]. Usual gait speed $(\mathrm{m} / \mathrm{s})$ on a $6 \mathrm{~m}$ course was used as an objective measure of physical performance and a slow walking speed was defined as a walking speed less than $0.8 \mathrm{~m} / \mathrm{s}[2]$.

\section{Statistical analysis}

SPSS21.0 was used to perform statistical analyses. Continuous variables were presented as mean \pm standard deviation $(\mathrm{M} \pm \mathrm{SD})$. Significance testing of the difference between the two groups was conducted using the independent samples t-test for continuous variables and the chi-square test or Fisher's exact test for categorical variables. Multiple logistic regression analysis was used to evaluate the association between risk factors and sarcopenia. A two-sided $p<0.05$ was considered statistically significant.

\section{Results}

Data from 834 participants were used for the analysis. The mean age was $68.57 \pm 6.44$ years (range: 6092 years) and $419(50.2 \%)$ participants were female. Of the 834 community-dwelling elderly Chinese people, $88(10.6 \%)$ participants were classified as having sarcopenia. For male, 23(48.9\%) of the participants with sarcopenia had low ASMI and low grip strength, 17(36.2\%) had low ASMI and low usual gait speed, 7(14.9\%) had both low muscle strength and low physical performance. These rates were 22(53.7\%), 19(46.3\%) and $0(0 \%)$ in women, respectively.

Table 1 presents the socioeconomic status and clinical characteristics of the participants by sex and sarcopenia status. Older participants were more likely to have sarcopenia than younger ones $(p<0.001)$. Participants with sarcopenia had statistically significantly lower BMI, ASM, ASMI, handgrip strength, and usual walking speed than participants without sarcopenia. History of cardiac diseases, hypertension, and stroke was more common in sarcopenic men than in nonsarcopenic participants.

Table 2 presents the lifestyle and family function score of participants by sex and sarcopenia status. Female participants who did not drink alcohol were more common in the sarcopenia group $(p<0.05)$. For men, the level of physical activity was significantly different between participants with or those without sarcopenia. Female participants with sarcopenia had statistically significantly lower frequency per week of nut consumption than those without sarcopenia. However, no statistically significant difference in regard to food items was observed between men with and those without sarcopenia. The mean Family APGAR score was $8(\mathrm{SD}=0.92)$. For both sexes, participants with sarcopenia had lower family function scores than those without sarcopenia. The prevalence rate of different levels of family function was significantly different in participants with or without sarcopenia for both sexes. 
Table 1 Baseline characteristics profile of participants

\begin{tabular}{|c|c|c|c|c|c|c|}
\hline & \multicolumn{3}{|c|}{ Male $(n=415)$} & \multicolumn{3}{|c|}{ Female $(n=419)$} \\
\hline & $\begin{array}{l}\text { Sarcopenia } \\
N=47\end{array}$ & $\begin{array}{l}\text { Non-sarcopenia } \\
N=368\end{array}$ & P & $\begin{array}{l}\text { Sarcopenia } \\
N=41\end{array}$ & $\begin{array}{l}\text { Non-sarcopenia } \\
N=378\end{array}$ & $P$ \\
\hline Age, yrs., Mean(SD) & $73.64(7.25)$ & $68.28(6.24)$ & $<0.001^{* *}$ & $72.85(6.98)$ & $67.76(6.03)$ & $<0.001^{* *}$ \\
\hline Weight, Kg, Mean(SD) & $59.01(10.75)$ & 64.92(9.00) & $<0.001^{* *}$ & $51.61(9.49)$ & $56.82(8.34)$ & $<0.001^{* *}$ \\
\hline Height,cm, Mean(SD) & $160.19(5.31)$ & $164.50(6.04)$ & $<0.001^{* *}$ & 151.22(6.09) & $152.99(5.96)$ & 0.071 \\
\hline BMI,kg/m2, Mean(SD) & $22.99(3.87)$ & $23.96(2.76)$ & $0.031^{*}$ & 22.52(3.71) & $24.26(3.15)$ & $0.001^{* *}$ \\
\hline Appendicular muscle mass, kg, Mean(SD) & $15.41(2.07)$ & $17.29(2.55)$ & $<0.001^{* *}$ & $11.64(1.17)$ & $13.33(1.98)$ & $<0.001^{* *}$ \\
\hline ASMI, kg/m2, Mean(SD) & $5.99(0.63)$ & $6.38(0.80)$ & $<0.001^{* *}$ & $5.09(0.46)$ & $5.69(0.72)$ & $<0.001^{* *}$ \\
\hline Grip strength, kg, Mean(SD) & $25.99(5.21)$ & $36.09(6.29)$ & $<0.001^{* *}$ & 17.53(3.19) & $22.99(4.46)$ & $<0.001^{* *}$ \\
\hline Usual gait speed, m/s, Mean(SD) & $0.86(0.26)$ & $1.09(0.17)$ & $<0.001^{* *}$ & $0.85(0.18)$ & $1.03(0.15)$ & $<0.001^{* *}$ \\
\hline \multicolumn{7}{|l|}{ Education years, n(\%) } \\
\hline 0 & $5(10.6)$ & $5(1.4)$ & $<0.001^{* *}$ & $6(14.6)$ & $9(2.4)$ & $<0.001^{* *}$ \\
\hline $1-6$ & 28(59.6) & $156(42.4)$ & & $25(61.0)$ & $145(38.4)$ & \\
\hline $7-9$ & $6(12.8)$ & $49(13.3)$ & & $5(12.2)$ & $57(15.1)$ & \\
\hline $10-12$ & $7(14.9)$ & 153(41.6) & & $5(12.2)$ & 159(42.1) & \\
\hline$>12$ & $1(2.1)$ & $5(1.4)$ & & 0 & $8(2.1)$ & \\
\hline \multicolumn{7}{|l|}{ Diseases, n(\%) } \\
\hline Cardiac diseases & $5(10.6)$ & 14(3.8) & $0.035^{*}$ & $1(2.4)$ & $17(4.5)$ & 0.537 \\
\hline Kidney disease & $1(2.1)$ & $4(1.1)$ & 0.538 & $0(0.0)$ & $7(1.9)$ & 0.380 \\
\hline Hypertension & $26(55.3)$ & 144(39.1) & $0.034^{*}$ & $21(51.2)$ & 156(41.3) & 0.221 \\
\hline Stroke history & $2(4.3)$ & $2(0.5)$ & $0.014^{*}$ & $0(0.0)$ & $4(1.1)$ & 0.508 \\
\hline Cancer & $0(0.0)$ & $2(0.5)$ & 0.612 & $0(0.0)$ & $2(0.1)$ & 0.541 \\
\hline Diabetes & $11(23.4)$ & $59(16.0)$ & 0.204 & $12(29.3)$ & $65(17.2)$ & 0.058 \\
\hline COPD & $1(2.1)$ & $3(0.8)$ & 0.386 & $0(0.0)$ & $7(1.9)$ & 0.380 \\
\hline MMSE scores & $26.6(1.9)$ & $27.1(2.4)$ & 0.079 & $25.4(4.0)$ & $26.3(3.2)$ & 0.088 \\
\hline GDS-15 scores & $1.6(1.3)$ & $1.5(1.7)$ & 0.780 & $1.8(1.9)$ & $1.5(1.9)$ & 0.414 \\
\hline
\end{tabular}

${ }^{*} p<0.05 ;{ }^{* *} p<0.01$ between non sarcopenic and sarcopenic individuals;BMI = body mass index; ASMI = appendicular skeletal muscle mass index; COPD $=$ chronic obstructive pulmonary disease. Using independent-samples $t$ test for continuous variables and Pearson chi-square or Fisher's exact test (where an expected cell count was $<5$ ) for categorical variables. During testing, $P<0.05$ was considered statistically significant

The association of lifestyle with sarcopenia is shown in Table 3. After adjustment for sex, age, educational levels, diabetes, hypertension, heart disease and stroke, a significant association was revealed between prevalence of sarcopenia and frequency per week of nut consumption (0.724, 95\% CI 0.532-0.985; $P<0.05)$. As for the other eight food items, no statistical significance was detected in either the unadjusted or adjusted models. In the unadjusted model, high level of physical activity (OR, 0.224; 95\% CI, 0.074-0.672; $P=0.008)$ and drinking $\geq 2$ times per week (OR, 0.435; 95\% CI, 0.206-0.919; $P=0.029)$ was found to be associated with sarcopenia. However, the effect disappeared after adjusting for other covariates. Multiple logistic regression models were also used to examine the association of family function with sarcopenia in Table 3. There was a significant association of Family APGAR scores with sarcopenia (0.683, 95\% CI $0.496-0.940, P=0.019)$.

\section{Discussion}

The present study was performed to evaluate the prevalence of sarcopenia among Chinese community-dwelling people aged $\geq 60$ years using the AWGS definition. Among the study population, $10.6 \%$ were diagnosed with sarcopenia. The prevalence of sarcopenia observed in this study was similar to the prevalence observed in Japanese and Korean populations, [22, 23] however, lower than that in Caucasian populations, [24, 25] This difference might partly originate from heterogeneity of study populations, but also due to the different techniques used to assess muscle mass.

Although sarcopenia can be explained by some fixed factors, many factors are not modifiable. This fact has led to increasing interest in the influence of lifestyle on muscle mass and function in older people. These factors include various aspects of food consumption patterns, physical activity, alcohol intake, and tobacco use. For 
Table 2 Lifestyle and family function of participants

\begin{tabular}{|c|c|c|c|c|c|c|}
\hline & \multicolumn{3}{|c|}{ Male $(n=415)$} & \multicolumn{3}{|c|}{ Female $(n=419)$} \\
\hline & $\begin{array}{l}\text { Sarcopenia } \\
N=47\end{array}$ & $\begin{array}{l}\text { Non-sarcopenia } \\
N=368\end{array}$ & $P$ & $\begin{array}{l}\text { Sarcopenia } \\
N=41\end{array}$ & $\begin{array}{l}\text { Non-sarcopenia } \\
N=378\end{array}$ & $P$ \\
\hline \multicolumn{7}{|l|}{ Smoking, n(\%) } \\
\hline Current Smokers & $14(29.8)$ & 106(28.8) & 0.413 & $0(0.0)$ & $9(2.4)$ & 0.445 \\
\hline Quitter & 15(31.9) & $151(41.0)$ & & $2(4.9)$ & $10(2.6)$ & \\
\hline Never Smokers & 18(38.3) & $111(30.2)$ & & 39(95.1) & 359(95.0) & \\
\hline \multicolumn{7}{|l|}{ Drinking, n(\%) } \\
\hline Not drink & $32(68.1)$ & 204(55.4) & 0.252 & $41(100.0)$ & $327(86.5)$ & $0.046^{*}$ \\
\hline Drinking <2/week & $6(12.8)$ & $61(16.6)$ & & $0(0.0)$ & $21(5.6)$ & \\
\hline Drinking $\geq 2 /$ week & $9(19.1)$ & $103(28.0)$ & & $0(0.0)$ & $30(7.9)$ & \\
\hline \multicolumn{7}{|l|}{ Present Physical activity, n(\%) } \\
\hline Low & $4(8.5)$ & $7(1.9)$ & $0.020^{*}$ & $1(2.4)$ & $6(1.6)$ & 0.387 \\
\hline Moderate & $20(42.6)$ & $141(38.4)$ & & 14(34.1) & $97(25.7)$ & \\
\hline High & $23(48.9)$ & 219(59.7) & & $25(61.0)$ & $275(72.7)$ & \\
\hline \multicolumn{7}{|c|}{ Dietary Pattern(times per week, Mean(SD)) } \\
\hline Grain,Cereals & $20.00(3.95)$ & $20.21(3.79)$ & 0.726 & $20.00(3.67)$ & $19.37(3.85)$ & 0.315 \\
\hline Vegetables & 14.98(12.61) & $17.12(11.66)$ & 0.242 & $18.22(11.11)$ & $16.90(11.92)$ & 0.498 \\
\hline Fruits & $5.11(3.90)$ & $5.22(3.71)$ & 0.840 & $5.39(3.29)$ & $6.44(3.53)$ & 0.071 \\
\hline Eggs & $5.36(3.02)$ & $4.86(2.89)$ & 0.263 & $4.32(2.62)$ & $4.73(2.72)$ & 0.355 \\
\hline Fish, shrimp & $1.04(1.49)$ & $0.85(1.29)$ & 0.353 & $0.98(1.54)$ & $0.90(1.30)$ & 0.744 \\
\hline Nuts & $0.19(0.74)$ & $0.61(1.90)$ & 0.135 & $0.05(0.22)$ & $0.81(2.11)$ & $0.022^{*}$ \\
\hline Meat(pork,beef,mutton,poultry) & $8.87(4.79)$ & $9.54(5.01)$ & 0.386 & $9.54(4.72)$ & $9.15(4.50)$ & 0.604 \\
\hline Milk,milk products & $4.87(3.40)$ & $4.45(4.45)$ & 0.532 & $3.86(3.14)$ & $4.68(3.17)$ & 0.112 \\
\hline legumes & $3.02(4.26)$ & $2.28(2.57)$ & 0.090 & $2.17(3.32)$ & $2.23(2.61)$ & 0.888 \\
\hline \multicolumn{7}{|l|}{ Family function } \\
\hline APGAR,Mean(SD) & $7.68(1.11)$ & $8.05(0.77)$ & $0.003^{* *}$ & $7.63(1.22)$ & $8.06(0.99)$ & $0.013^{*}$ \\
\hline Good family function & $40(85.1)$ & $355(96.5)$ & $0.001^{* *}$ & $35(85.4)$ & $363(96.0)$ & $0.004^{* *}$ \\
\hline Moderate family dysfunction & $7(14.9)$ & $12(3.2)$ & & $5(12.2)$ & $9(2.4)$ & \\
\hline severe family dysfunction & $0(0.0)$ & $1(0.3)$ & & $1(2.4)$ & $6(1.6)$ & \\
\hline Live along, n(\%) & $4(8.5)$ & $19(5.2)$ & 0.345 & $8(19.5)$ & $48(12.7)$ & 0.088 \\
\hline
\end{tabular}

${ }^{*} p<0.05 ;{ }^{* *} p<0.01$ between non sarcopenic and sarcopenic individuals. During testing, $P<0.05$ was considered statistically significant

older adults, altered taste and smell, social changes, and economic limitations may also lead to decreased food intake, [26] especially a low nutrient intake. Inadequate intake of nutrients is one of the major mechanisms underlying sarcopenia. Recent studies have demonstrated that an intake of nutrients, in particular, protein intake, EAA, and Vitamin D, have an influence on skeletal muscle metabolism [4-6]. In this study, after adjustment for sex, ages, educational levels, diabetes, hypertension, heart disease and stroke, the frequency per week of nut consumption was significantly associated with sarcopenia (0.724, 95\% CI $0.532-0.985 ; P<0.05)$. An adequate intake of nuts may be a protective factor against sarcopenia. Most of the participants in the study had a diet inclusive of nuts, including peanuts, walnut kernels or cashews, which abound in EAA such as leucine, valine, methionine and tryptophan. Recent studies have demonstrated that the leucine attenuated skeletal muscle wasting occurs by an interaction with proteolytic pathways [5]. Katsanos et al. has shown that increasing the proportion of leucine in a mixture of EAA given to elderly subjects can reverse the attenuated response of muscle protein synthesis [27]. An adequate intake of nuts that are abundant in leucine may serve as a potential strategy to combat the progression of sarcopenia. Our study provided supporting evidence about the association of the intake of nutrients and sarcopenia.

Chronic alcohol intake and current smoking are other lifestyle habits that have been associated with sarcopenia in previous studies [7, 8]. In the present study, after adjustment for sex, ages, educational levels, diabetes, 
Table 3 Unadjusted and adjusted model for factors related to Sarcopenia

\begin{tabular}{|c|c|c|}
\hline Factors & $\begin{array}{l}\text { Unadjusted } \\
\text { OR(95\% CI) }\end{array}$ & $\begin{array}{l}\text { Adjusted } \\
\text { OR(95\% Cl) }\end{array}$ \\
\hline \multicolumn{3}{|l|}{ Dietary pattern } \\
\hline Grain, Cereals & $1.015(0.956-1.078)$ & $1.018(0.952-1.089)$ \\
\hline Vegetables & 0.998(0.979-1.017) & $1.001(0.980-1.023)$ \\
\hline Fruits & $0.956(0.895-1.022)$ & $0.961(0.888-1.039)$ \\
\hline Eggs & $1.007(0.932-1.088)$ & $0.996(0.914-1.087)$ \\
\hline Fish,shrimp & $1.061(0.913-1.232)$ & $1.012(0.866-1.181)$ \\
\hline Nuts & $0.702(0.516-0.956)^{*}$ & $0.724(0.532-0.985)^{*}$ \\
\hline Meat & 0.993(0.946-1.042) & $1.000(0.948-1.054)$ \\
\hline Milk, milk products & 0.990(0.932-1.052) & $0.957(0.883-1.037)$ \\
\hline Legumes & $1.034(0.962-1.112)$ & $1.016(0.941-1.098)$ \\
\hline \multicolumn{3}{|l|}{ Physical activity } \\
\hline Low & 1 (reference) & 1 (reference) \\
\hline Moderate & $0.337(0.111-1.026)$ & $0.407(0.093-1.779)$ \\
\hline High & $0.224(0.074-0.672)^{*}$ & $0.372(0.085-1.634)$ \\
\hline \multicolumn{3}{|l|}{ Smoking } \\
\hline Never Smoker & 1 (reference) & 1 (reference) \\
\hline Current smokers & $1.137(0.630-2.053)$ & $1.365(0.585-3.186)$ \\
\hline quitter & $0.773(0.426-1.403)$ & $0.637(0.286-1.423)$ \\
\hline \multicolumn{3}{|l|}{ Drinking } \\
\hline Not drink & 1 (reference) & 1 (reference) \\
\hline Drinking <2/week & $0.530(0.218-1.294)$ & $0.610(0.235-1.579)$ \\
\hline Drinking $\geq 2 /$ week & $0.435(0.206-0.919)^{*}$ & $0.527(0.233-1.192)$ \\
\hline APGAR & $0.699(0.576-0.848)^{*}$ & $0.683(0.496-0.940)^{*}$ \\
\hline
\end{tabular}

Binary logistic analyses were used to estimate the $\mathrm{OR}$ and $95 \% \mathrm{Cl}$ of factors related to sarcopenia; Adjusted for gender, ages, educational levels,diabetes, hypertension, heart disease, stroke, MMSE score and GDS score ${ }^{*} P<0.05$

hypertension, heart disease, and stroke, no significant difference was revealed between sarcopenia and these habits. This variance might be due to the type of questions regarding these habits on the questionnaire, which did not specify quantity or frequency of smoking or alcohol consumption, so we could not conclude whether or not there was an excess of alcohol and tobacco consumption in these participants. Therefore, the association between smoking and alcohol intake with sarcopenia should be further investigated. In the multivariate model, physical activity was not significantly associated with sarcopenia. Previous study showed resistance training was demonstrated to be more effective in attenuating the development of sarcopenia $[9,28]$. The conflicting results may be explained for the different physical activities. Because in the study participants only took part in some baseline physical activities, which may be inactive compared with other strength training programs.
For most Chinese elderly, families are the primary source of social support, which is a recognized determinant of health [29]. Several studies found family function affected the health conditions and quality of the care and services for the aged with some chronic diseases [30]. If older adults enjoy a well-functioning family life or family support, their health functions will also enable them to enjoy good daily functioning and self-care ability [12]. However, presently few studies have focused on the relationship between family function and sarcopenia. To the best of our knowledge, this was the first research study that has focused on the association between family function and sarcopenia. In this study, after adjusting for age, sex, and education level, and medical disease, a significant association was found between family function score and sarcopenia. However, the latent basis of the association between family function and sarcopenia is uncertain. Family function is associated with psychological outcomes, metabolic control, and eating behaviors or dietary patterns in the elderly. These may be possible mechanisms to consider as an underlying basis of this association. Further studies should evaluate if good family function may be effective in lowering the risk of sarcopenia.

This study has several strengths. First, it is one of the studies in Asia to use the Asian Working Group for Sarcopenia criteria in Asia. Second, the study was conducted using a relatively large sample in China, which was a well-characterized population of community-dwelling older adults living in a defined geographical area. Our sample included participants that ranged in age from 60 to 92 years. There were several limitations of our study. First, the assessment of lifestyle and family function was based on cross-sectional measures, so it was not possible to determine causal relationships. Second, only ambulatory participants were surveyed because participants who were in hospitals or had serious diseases were not included.

\section{Conclusion}

In conclusion, we found the prevalence of sarcopenia among community-dwelling Chinese population aged 60 years and older was high and there was significant association between sarcopenia and intake of nuts and family function.

\section{Abbreviations}

ASM: Appendicular skeletal muscle mass; ASMI: Appendicular skeletal muscle mass index; AWGS: Asian Working Group for Sarcopenia; BIA: Bioelectrical impedance analysis; BMI: Body mass index; EWGSOP: European Working Group on Sarcopenia in Older People; GS: Gait speed; HS: Handgrip strength

\section{Acknowledgments}

The authors thank the staff of the Department of Geriatrics, West China Hospital, and all the participants (as well as their legal proxies) for their great contribution. 


\section{Funding}

This work was supported by grants from the Project of Benefit the People of the Technology Bureau in Chengdu: To Establish the Service Standard of Home-care System (2015-HM02-00024-S).

\section{Availability of data and materials}

The datasets generated during and/or analysed during the current study available from the corresponding author on reasonable request.

\section{Authors' contributions}

$B R D, S H, H W$ and $L C$ designed the study. HW, SH, LC, JHZ, PL and YY recruited the subjects and collected the data. HW and LC performed statistical analyses. $\mathrm{SH}$ drafted the manuscript. All authors read and approved the final manuscript.

\section{Ethics approval and consent to participate}

The study protocol was approved by the Research Ethics Committee of Sichuan University with the following reference number (2014, No.57). All participants (and their legal proxies) provided written informed consent.

\section{Consent for publication}

Not applicable.

\section{Competing interests}

No author of this paper has any conflict of interests related to this study.

\section{Publisher's Note}

Springer Nature remains neutral with regard to jurisdictional claims in published maps and institutional affiliations.

Received: 6 December 2016 Accepted: 14 August 2017

Published online: 18 August 2017

\section{References}

1. Delmonico MJ, Harris TB, Visser M, Park SW, Conroy MB, Velasquez-Mieyer P, et al. Longitudinal study of muscle strength, quality, and adipose tissue infiltration. Am J Clin Nutr. 2009:90:1579-85.

2. Chen LK, Liu LK, Woo J, Assantachai P, Auyeung TW, Bahyah KS, et al. Sarcopenia in Asia: consensus report of the Asian working Group for Sarcopenia. JAMDA. 2014;15:95-101.

3. Burton LA, Sumukadas D. Optimal management of sarcopenia. Clin Interv Aging. 2010;7:217-28.4

4. Kim JS, Wilson JM, Lee SR. Dietary implications on mechanisms of sarcopenia: roles of protein, amino acids and antioxidants. J Nutr Biochem. 2010;21:1-13.

5. Nicastro H, Artioli GG, Costa Ados S, Solis MY, da Luz CR, Blachier F, et al. An overview of the therapeutic effects of leucine supplementation on skeletal muscle under atrophic conditions. Amino Acids. 2011:40:287-300.

6. Scott D, Blizzard L, Fell J, Ding C, Winzenberg T, Jones G. A prospective study of the associations between 25-hydroxyvitamin D,sarcopenia progression, and physical activity in older adults. Clin Endocrinol. 2010;73:581-7.

7. Preedy VR, Adachi J, Ueno Y, Ahmed S, Mantle D, Mullatti N, et al. Alcoholic skeletal muscle myopathy: definitions, features, contribution of neuropathy, impact and diagnosis. Eur J Neurol. 2001;8:677-87.

8. Lee JS, Auyeung TW, Kwok T, Lau EM, Leung PC, Woo J. Associated factors and health impact of sarcopenia in older Chinese men and women: a crosssectional study. Gerontology. 2007:53:404-10.

9. Eibich P, Buchmann N, Kroh M, Wagner GG, Steinhagen-Thiessen E, Demuth I, et al. Exercise at different ages and appendicular lean mass and strength in later life: results from the berlin aging study II. J Gerontol A Biol Sci Med Sci. 2016;71:515-20.

10. Kim SH, Kim TH, Hwang HJ. The relationship of physical activity (PA) and walking with sarcopenia in Korean males aged 60 years and older using the fourth Korean National Health and nutrition examination survey (KNHANES IV-2, 3), 2008-2009. Arch Gerontol Geriatr. 2013;56:472-7.

11. Baumgartner RN, Koehler KM, Gallagher D, Romero L, Heymsfield SB, Ross RR, et al. Epidemiology of sarcopenia among the elderly in New Mexico. Am J Epidemiol. 1998:147:755-63.

12. Wang MW, Huang YY. Evaluating family function in caring for a geriatric group: family APGAR applied for older adults by home care nurses. Geriatr Gerontol Int. 2016;16:716-21.
13. Wang B, He P, Dong B. Association between family functioning and cognitive impairment among Chinese nonagenarians/centenarians. Geriatr Gerontol Int. 2015;15:1135-42.

14. Seeman TE. Health promoting effects of friends and family on health outcomes in older adults. Am J Health Promot. 2000;14:362-70.

15. Tombaugh TN, Mclntyre NJ. The mini- mental state exam: a comprehensive review. J Am Geriatr Soc. 1992:40:922-35.

16. Tang D. Application of short form geriatric depression scale (GDS-15) in Chinese elderly. J Chinese Clin Psy. 2013;21:402-5.

17. Zhang CX, Ho SC. Validity and reproducibility of a food frequency questionnaire among Chinese women in Guangdong province. Asia Pac J Clin Nutr. 2009;18: 240-50

18. Hallal PC, Victora CG. Reliability and validity of the International Physical Activity Questionnaire (IPAQ). Med sci sport exer. 2004;36:556.

19. Smilkstein G. The family APGAR: a proposal for a family function test and its use by physicians. J Fam Pract. 1978;6:1231-9.

20. Lv F, Zeng G, Liu SN, Zhong TL, Zhan ZQ. A study on validity and reliability of the family APGAR. Chin Public Health (Chinese). 1999;15:987-8.

21. Smilkstein G, Ashworth C, Montano D. Validity and reli- ability of the family APGAR as a test of family function. J Fam Pract. 1982;15:303-11.

22. Tanimoto $Y$, Watanabe M, Sun W, Sugiura Y, Hayashida I, Kusabiraki T, et al. Sarcopenia and falls in community-dwelling elderly subjects in Japan: defining sarcopenia according to criteria of the European working group on sarcopenia in older people. Arch Gerontol Geriatr. 2014:59:295-9.

23. Lin CC, Lin WY, Meng NH, Li Cl, Liu CS, Lin CH, et al. Sarcopenia prevalence and associated factors in an elderly Taiwanese metropolitan population. Am Geriatr Soc. 2013;61:459-62

24. Cuesta F, Formiga F, Lopez-Soto A, Masanes F, Ruiz D, Artaza I, et al. Prevalence of sarcopenia in patients attending outpatient geriatric clinics: the ELLI study. Age Ageing. 2015;44:807-9.

25. Spira D, Norman K, Nikolov J, Demuth I, Steinhagen-Thiessen E, Eckardt R. Prevalence and definition of sarcopenia in community dwelling older people : data from the berlin aging study II (BASE-II). Z Gerontol Geriatr. 2016:49:94-9.

26. Buford TW, Anton SD, Judge AR, Marzetti E, Wohlgemuth SE, Carter CS, et al. Models of accelerated sarcopenia: critical pieces for solving the puzzle of age-related muscle atrophy. Ageing Res Rev. 2010;9:369-83.

27. Katsanos CS, Kobayashi H, Sheffield-Moore M, Aarsland A, Wolfe RR. A high proportion of leucine is required for optimal stimulation of the rate of muscle protein synthesis by essential amino acids in the elderly. Am J Physiol Endocrinol Metab. 2006;291:381-7.

28. Peterson MD, Sen A, Gordon PM. Influence of resistance exercise on lean body mass in aging adults: a meta-analysis. Med Sci Sports Exerc. 2011;43: 249-58.

29. Oliveira SC, Pavarini SCl, Orlandi FS, Mendiondo MS. Family func- tionality: a study of Brazilian institutionalized elderly indi- viduals. Arch Gerontol Geriatr. 2014;58:170-6.

30. Kamen C, Cosgrove V, McKellar J, Cronkite R, Moos R, et al. Family support and depressive symptoms: a 23-year follow-up. J Clin Psychol. 2011;67:215-23.

\section{Submit your next manuscript to BioMed Central and we will help you at every step:}

- We accept pre-submission inquiries

- Our selector tool helps you to find the most relevant journal

- We provide round the clock customer support

- Convenient online submission

- Thorough peer review

- Inclusion in PubMed and all major indexing services

- Maximum visibility for your research

Submit your manuscript at www.biomedcentral.com/submit
C) Biomed Central 